\title{
EVALUATION OF THE IMPACTOF DYNAMIC WORK STATIONS VERSUS STATIC WORK STATIONS IN WOOD FRAMING PREFABRICATION USING HYBRID SIMULATION
}

\author{
Beda BARKOKEBAS ${ }^{1,4}$, Samer BU HAMDAN ${ }^{2}$, Aladdin ALWISY ${ }^{2}$, Mohamed AL-HUSSEIN ${ }^{3}$ \\ ${ }^{1}$ MSc student, Department of Civil Engineering University of Alberta \\ ${ }^{2}$ PhD student, Department of Civil Engineering University of Alberta \\ ${ }^{3}$ Professor, Department of Civil Engineering University of Alberta \\ ${ }^{4}$ Corresponding author's e-mail: barkokeb@ualberta.ca
}

\begin{abstract}
Offsite manufacturing has introduced significant improvements in terms of both time and cost savings to the construction industry. The fabrication of modular units and construction components in factories has permitted the reshaping of the traditional stick-built process. By reallocating the majority of onsite activities to offsite facilities, onsite preparation tasks can be performed concurrently to the offsite production. The success of offsite manufacturing relies on the efficiency of the factory's production line. Continuous workflow improves factory efficiencies by reducing or eliminating fluctuations and bottlenecks among work stations. Imbalance in the production line is a result of work station capacity errors and other conditions unique to the construction industry. Unlike other industries, construction projects are often customized and have lower repetition quantities. The variations in the modular units or components being produced poses a challenge in balancing traditional work stations along the production line due to continuous changes in complexity level, which in turn affects productivity. This research proposes the use of dynamic work stations along with traditional ones, using multi-skilled workers relocating among specific work stations in response to product complexity levels. Two approaches are evaluated in order to balance the production line: (1) increase number of workers in static work stations; and (2) use dynamic work stations. A production comparison is performed using a hybrid simulation model, combining discrete-event and continuous simulation. The plotted results identify the optimum number of workers in the two stations, static versus dynamic, to meet demand. The model is validated and is found to achieve a reduction of $18.68 \%$ and $32.00 \%$ in the total production time for two different scenarios without increasing the original number of workers.
\end{abstract}

Keywords: Offsite Manufacturing, Dynamic Work Station, Discrete-Event Simulation, Continuous Simulation, Multi-skilled workers.

\section{INTRODUCTION}

Modular construction is widely utilized in North America, providing an efficient system to build structures quickly in a controlled environment (Mohsen et al. 2008; Olearczyk et al. 2014). Offsite construction has revolutionized the industry by presenting a new approach that divides work into two phases: offsite and onsite. In offsite building, components are manufactured in a controlled environment that permits increased quality and reduces cycle time. Offsite manufacturing has revolutionized the construction industry by introducing innovative manufacturing concepts. This has created opportunities for enhanced efficiency and competitiveness, and has actualized mass production to the construction industry.

Although it offers remarkable advantages, offsite construction requires thorough planning of operations and tasks related to production line, inventory, supply chain, and so forth. The backbone of the manufacturing process is the production line, where building materials are used to fabricate the required components. All activities on the production line are critical in order to achieve maximum efficiency; bottlenecks, such as idle stations, must be avoided so that continuous workflow is ensured. Moghadam (2014) has pointed out that due to the range of variation in modules (size, structure, layout, features, etc.) there is high variability in production rate in construction manufacturing, which affects the entire 
production line. This highlights the need to design a framework that, despite these variations, is still able to plan the production phases with minimal changes.

One suitable solution is the introduction of multi-skilled labour in the production line; instead of working only in one specific station, the worker can work in multiple stations in order to balance the production rate depending on the actual production sequence of the modules. However, hiring and maintaining a multi-skilled employee can prove to be a difficult task involving such challenges as shortage of available workers and increased training costs, wages, and turnover.

Computer-based simulation has proven to be an efficient approach to evaluate, predict, and forecast construction processes (AbouRizk and Hajjar 1997). Several studies have been published using discrete event simulation (DES) in modular manufacturing to assess various risk factors (Li et al. 2014) and system efficiency (Moghadam 2014; Hammad et al. 2002). Brito et al. (2011) have indicated that a combination of DES and continuous simulation (CS), referred to as hybrid simulation, is potentially the optimal approach for simulation, particularly when the simulation requires both a low level of abstraction (e.g., predicting a start and end to a task provided by DES) and a higher level of abstraction (e.g., a dynamic change to the production rate of an activity due to an increase or decrease of resources).

This paper presents a model that combines DES and CS in order to evaluate the impact of multiskilled labour on the production rate of the entire process. It also provides decision makers with information to evaluate the need to employ multi-skilled workers in order to achieve significant efficiency in the production line.

\section{METHODOLOGY}

The main objective of the proposed research is to evaluate the effect of incorporating dynamic work stations as compared to the traditional usage of static work stations in order to achieve a capacity buffer that is able to balance the fluctuations of workflow due to variations in the complexity level of tasks. In the context of this paper, the traditional static work station is defined as a work station with a fixed number of workers capable of performing specific tasks. In contrast, the dynamic work station comprises multi-skilled workers capable of performing tasks required in two or more stations. For the purpose of simplifying the analysis, the multi-skilled worker can perform tasks only in two sequential stations.

In industrialized construction, components are produced in a factory, being fabricated in a sequence of stations, and are shipped either as wall and floor panels or as modular units. The proposed simulation engine models the production line process with a focus on two stations. The size of the crew working at each station, the quantity of panels produced daily, and the size and complexity of the panels are all key input factors in the simulation engine (see Figure 1). The collected data from Star Prebuilt Homes, the case study manufacturing plant, is used to evaluate two approaches. The first approach is two static work stations, where a worker stands idle until the product from the previous station is transferred to their station. If the complexity of the previous station is high, delays will occur and cause a bottleneck. In this approach, an increase in number of workers is proposed to improve the product flow. The second approach is two hybrid (static and dynamic) work stations, where the required labour to balance the production line in order to improve workflow and reduce fluctuation is employed as a function of the dynamic work station. Once the task is complete in one station, the multi-skilled worker returns to their original station. Figure 1 illustrates the proposed methodology that evaluates the two approaches and identifies the ideal number of multi-skill workers in order to achieve the required capacity buffer in the production line. 


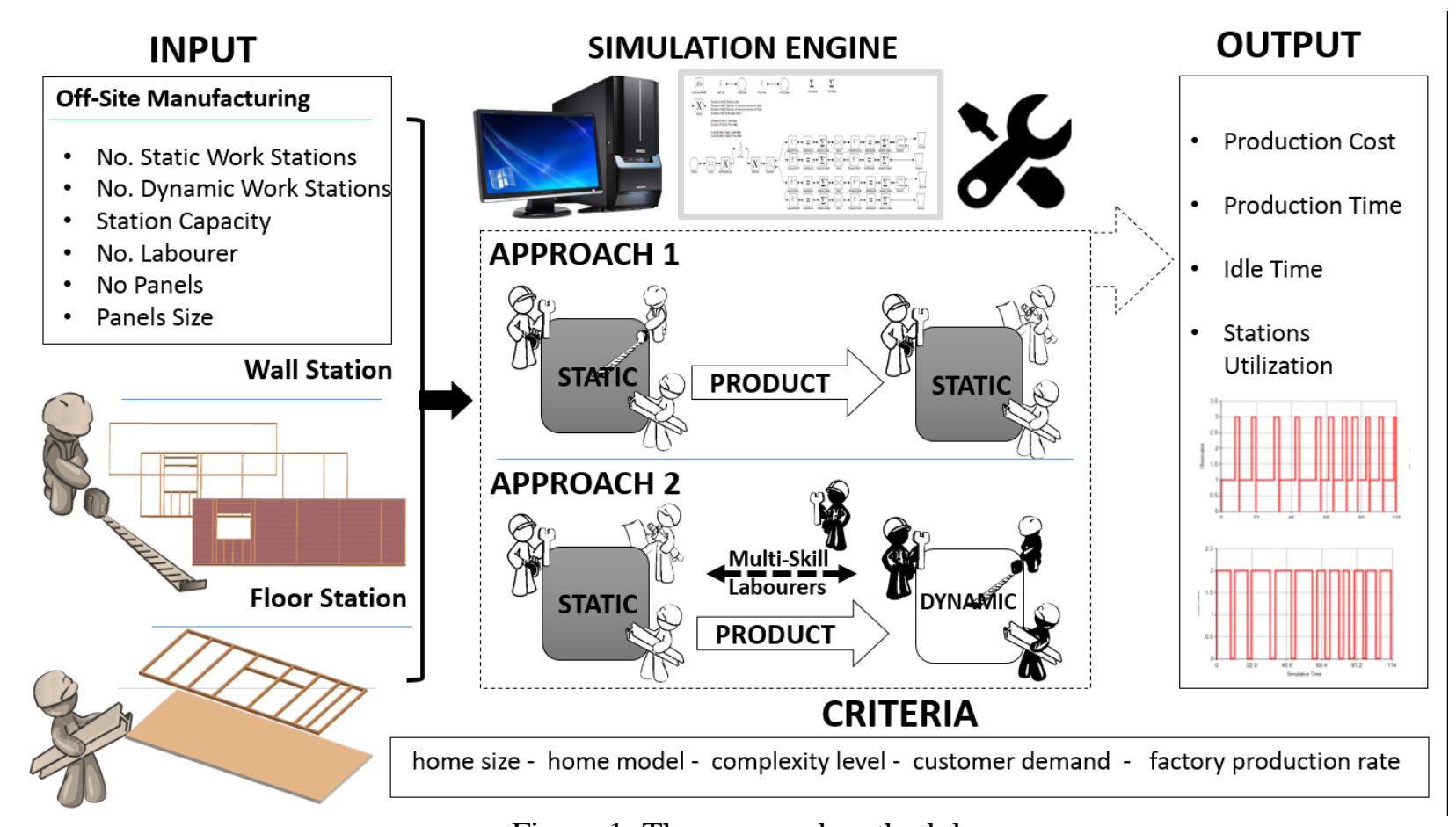

Figure 1: The proposed methodology.

The two stations being evaluated (wall and floor framing) are chosen from a total of sixteen labour stations since both are predecessors of the cubing station (assembly of framed walls on the floor), and since workers assigned to both stations receive similar training (wood framing) and are able to perform the tasks at both stations. Figure 2 describes the evaluated process when the work initiates in Stations OA (one worker) and OB (two workers) at time 0, and should be conveyed to Station 1 for assembling. Originally, two and one workers are assigned to stations $0 \mathrm{~A}$ and $0 \mathrm{~B}$ respectively.

Time: 0

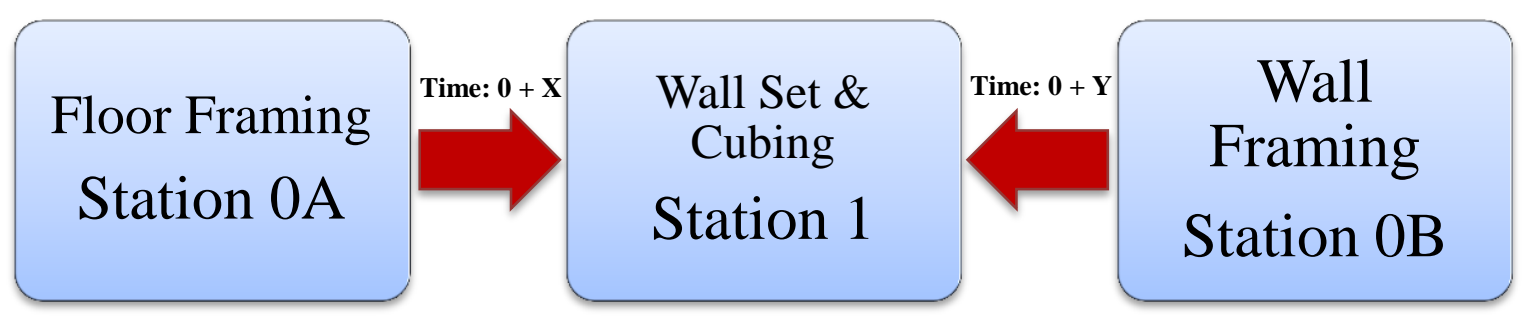

Figure 2: Current set of stations

However, due to a large number of potential module variations (size, features, etc.) and different production rates between stations, durations ( $X$ and $Y$ ) for each station vary with a fixed number of workers, creating common delays between stations since workers are unable to move the finished module and cannot initiate a new module due to space limitations. The proposed simulation thus aims to reduce the difference between $X$ and $Y$ by adding workers, when idle, to the delayed work stations $(0 \mathrm{~A}$ or $\mathrm{OB})$ and to thereby increase its productivity within a limited time, simulating a brief participation of multi-skilled workers when needed. 


\subsection{Simulation model}

To simulate the operation, a model is developed in a Simphony.NET 4.0 environment, as demonstrated in Figures 3 and 4, showing DES and CS components, respectively. The main focus of the model is to evaluate the impact of cross-training on the productivity of the entire system. At the DES component, the modules are created with the hour requirements for each activity, and this requires the resources to be initially available at each station. Once resources are captured (two resources for the floor station and one for the wall station), the entity is held until the proposed activity is started and terminated at the CS component.

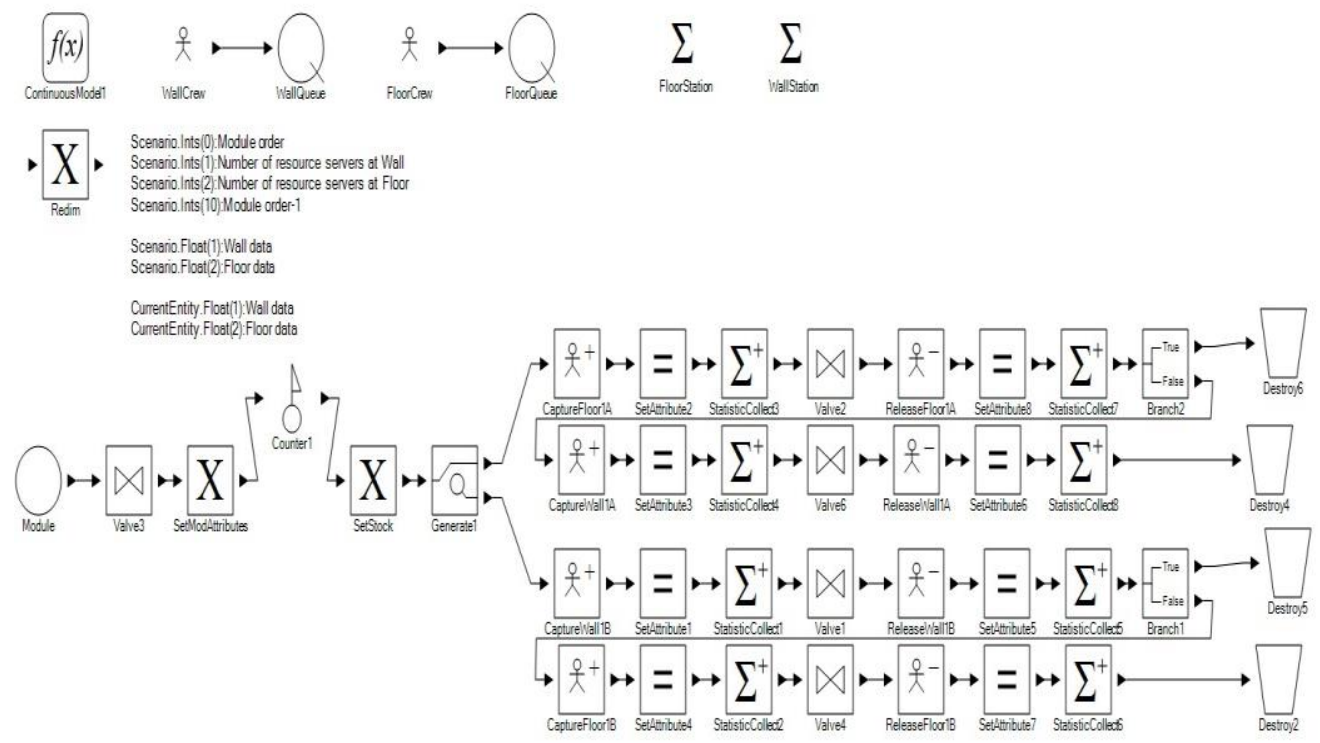

Figure 2: DES component of simulation model.

In the CS component, the stocks for each station are created with the same value as the hour requirements in the DES component, and the activity is performed with its rate (i.e., labour production rate) according to the number of captured resources. After completion of the first activity, the entity is released in the DES component and captures the resource in order to help the delayed station by increasing its production rate (cross-training between stations).
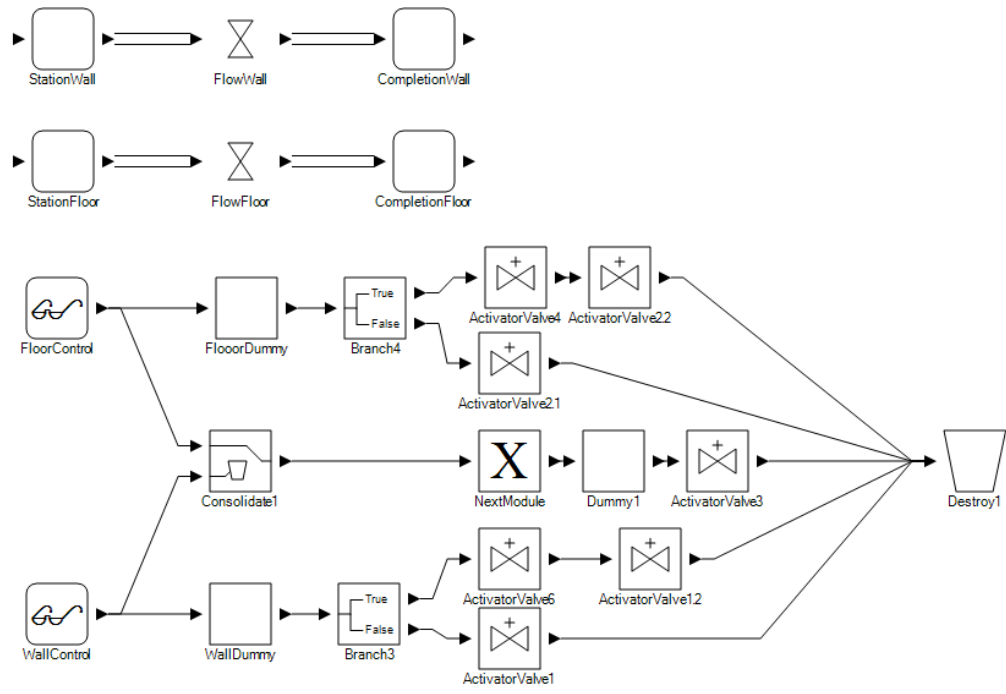

Figure 3: CS component of simulation model. 
When the task at the wall station finishes, the entity captures one extra floor resource, which increases the production rate by one extra worker. The floor station, when the activity is terminated, captures only one resource (two workers) due to space limitations in the factory. The process is repeated until there are no more modules available. The model provides as output the mean and peak number of workers and a histogram that tracks the resources in each respective station for the duration of the process.

\section{MODEL VALIDATION}

In order to validate the model, data for wall and floor stations of eleven different modules is taken from Moghadam's study (2014) and simulated in the proposed model without cross-training between work stations. After completion of its station, the model requires no resources to increase the production rate of the next station. The durations are obtained through an extensive study of modular manufacturing and, as part of the research, a methodology is created to predict the hour requirements for several activities of the production line taking into consideration several factors such as size, features, and layout of modules. Running the model with the same labour conditions as stated (no cross-training), the total time for completion of the eleven modules is found to be 168.6 hours, a difference of $0.9 \%$ if compared to the result (167.0 hours) found previously in Moghadam's study (2014).

\section{SIMULATION RESULTS}

After the validation process, the model is set to a cross-training scenario: after a task is completed at one station, the station lends one worker to work in another station when needed. From the simulation results, two scenarios are generated as displayed in Figure 4. The total time for Scenario 1 is reduced to 137.10 hours, a reduction of $18.68 \%$ over the current state without cross-training; the resource allocation is displayed in Figure 4.
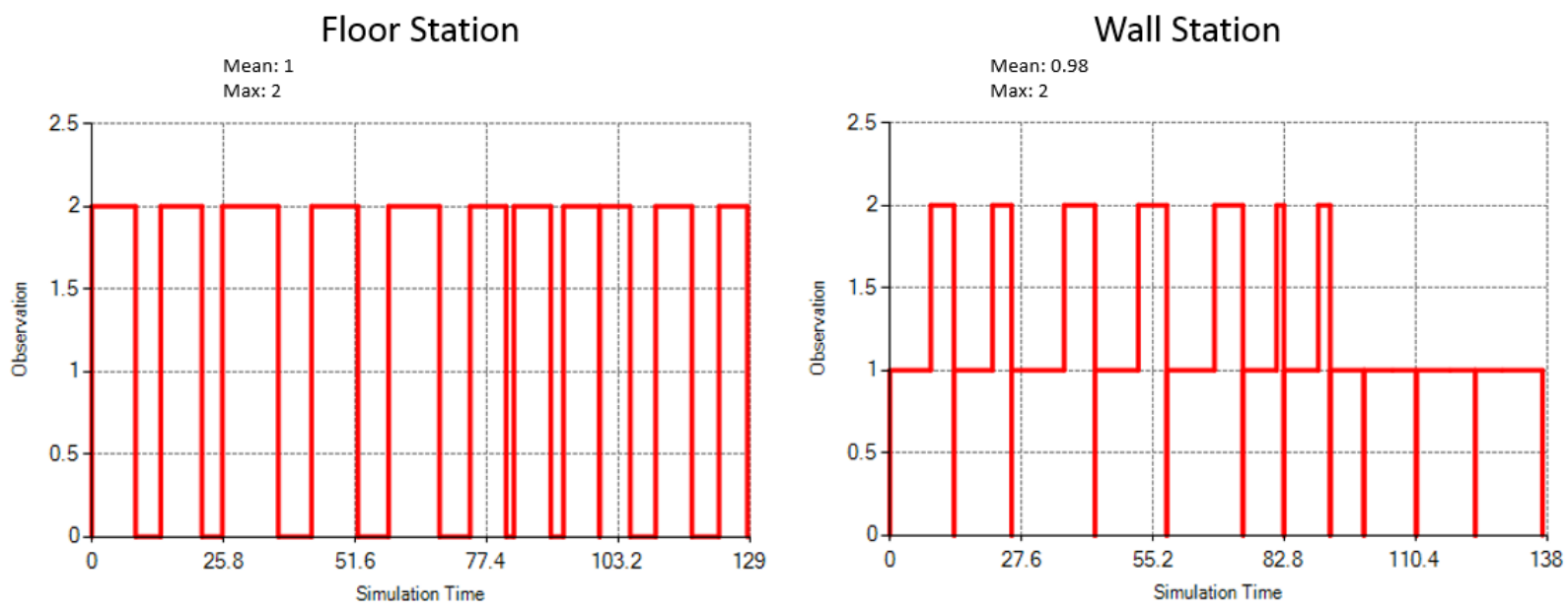

Figure 4: Station resource allocation for Scenario 1.

As observed in Figure 4, the floor station is found to finish tasks early in all instances, sending one worker to the other station in order to help balance the work. As stated by its peak of two (maximum number of workers working on that station) and its mean of one, the floor station does not require any help from the wall station; however, it can possibly reduce the number of workers by one. Figure 4 also indicates that the wall station requires help from workers from another station on seven out of eleven occasions and generates a mean utilization of 0.98 . This indicates that the number of fixed workers for this station is suitable since its utilization mean (0.98) is close to the assigned number (one). However, in some instances help is required from workers from other stations, as 
demonstrated in Figure 4, and as indicated by its peak utilization of two.

According to simulation results from Scenario 1, the suitable number of workers at the floor station is one, instead of two as currently assigned. The wall station contains the appropriate number of workers, but does require two workers working simultaneously. Scenario 2 is assembled with floor and wall stations having one and two workers, respectively. This configuration is created with the intent to let the wall station control the overall production since the floor station does not have a space limitation and can accommodate three workers at the same time (one from floor station plus two from wall station).
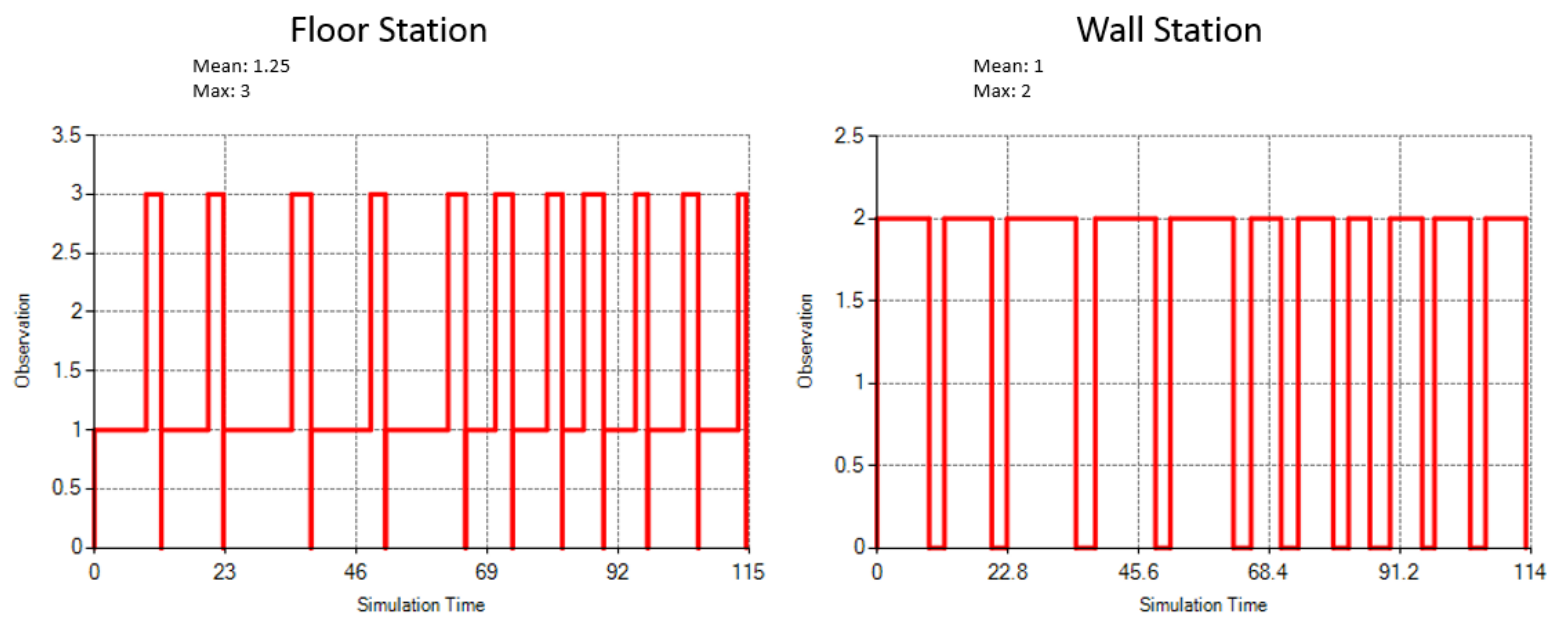

Figure 5: Station resource allocation for Scenario 2.

The total simulation time for Scenario 2 is found to be 114.58 hours, which is a reduction of $32.0 \%$ from the current scenario. Figure 5 demonstrates the resource allocation for Scenario 2, showing that the floor station receives help from wall station workers in all instances and that the second station controls the overall production. In Scenario 2, the allocation of one fixed worker for each of the stations is appropriate (means of 1.25 and 1, respectively), but the use of a multi-skilled worker is fundamental for the optimal performance of the entire system.

\section{CONCLUSION}

Managing human resources is a concern in the manufacturing industry, as it affects productivity as well as profit. Offsite construction is no exception to this rule. Having more resources than is required at each station can reduce productivity and increase cost. Additionally, management has to consider the skill level of each worker; therefore, management must balance the crew size at each station according to labour skill level in order to improve performance. It is clear from this research that the use of multiskilled workers can bring significant improvement to overall production without increasing the number of workers in the production line. While hiring low-skilled labour is inexpensive, the productivity may be compromised; high-skilled labour, on the other hand, is more expensive, but it is more productive. Therefore, a company must hire both high-skilled and low-skilled labour, assigning low-skilled labour statically to some stations, allocating high-skilled personnel dynamically to stations in a manner that allows them to work in multiple stations according to their skills. This pattern of resource allocation improves productivity, while saving time and cost and reducing delays in product completion. 


\section{ACKNOLEDGMENTS}

This research is conducted by the construction research group at the University of Alberta, in collaboration with Star Prebuilt Homes. The authors wish to thank the Natural Sciences and Engineering Research Council of Canada (NSERC) for financial support.

\section{REFERENCES}

Abourizk, S. M., and Hajjar, D. (1998). "A framework for applying simulation in construction." Canadian Journal of Civil Engineering, 25(3), 604-617.

Brito, T., Trevisan, E. F. C., and Botter, R. C. (2011). "A conceptual comparison between discrete and continuous simulation to motivate the hybrid simulation methodology." Proceedings of the 2011 Winter Simulation Conference, Phoenix, AZ, USA, Dec. 11-14.

Czarnecki, H., Schroer, B. J., and Rahman, M. M. (1997). "Using simulation to schedule manufacturing resources." Proceedings of the 1997 Winter Simulation Conference, Atlanta, GA, USA, Dec. 7-10.

Li, H. X., Al-Hussein, M., Lei, Z., and Ajweh, Z. (2013). "Risk identification and assessment of modular construction utilizing fuzzy analytic hierarchy process (AHP) and simulation." Canadian Journal of Civil Engineering, 40(12), 1184-1195.

Moghadam, M. (2014). "Lean-Mod: An approach to modular construction manufacturing (MCM) production efficiency improvement." $\mathrm{PhD}$ thesis, University of Alberta, Edmonton, AB, Canada.

Moghadam, M., Al-Hussein, M., Al-Jibouri, S., and Telyas, A. (2012). "Post simulation visualization model for effective scheduling of modular building construction." Canadian Journal of Civil Engineering, 39(9), 1053-1061.

Mohsen, O., Knytl, P., Abdulaal, B., Olearczyk, J., and Al-Hussein, M. (2008). "Simulation of modular building construction." Proceedings of the 2008 Winter Simulation Conference, Miami, FL, USA, Dec. 7-10.

Olearczyk, J., Al-Hussein, M., and Bouferguene, A. (2014). "Evolution of the crane selection and onsite utilization process for modular construction multilifts." Automation in Construction, 43, 59-72. 